\title{
Preparations of diversely substituted thiosemicarbazides and $N$-hydroxythioureas
}

\author{
Alan R. Katritzky*, Niveen M. Khashab, and Anna V. Gromova \\ Center for Heterocyclic Compounds, University of Florida, Department of Chemistry, \\ Gainesville, Florida 32611-7200, USA \\ E-mail: Katritzky@chem.ufl.edu
}

Dedicated to Prof. James Coxon on his $65^{\text {th }}$ birthday

\begin{abstract}
Thiosemicarbazides 5 (yields 50-97\%) and $N$-hydroxythioureas 6 (yields 71-99\%) of variable substitution patterns are prepared efficiently by reactions of 1-(thiocarbamoyl)benzotriazoles $\mathbf{4 a - i}$ with hydrazines or hydroxylamines, respectively.
\end{abstract}

Keywords: Thiosemicarbazides, $N$-hydroxythioureas, isothiocyanates

\section{Introduction}

\section{Thiosemicarbazides}

Thiosemicarbazides are valuable building blocks for the synthesis of five-membered heterocycles. ${ }^{1}$ Biologically active thiosemicarbazide derivatives include 1,3,4-thiadiazoles, as antibacterial $^{2}$ and antifungal ${ }^{3}$ agents, and 1,3,4-thiadiazolium-2-amidines as anticonvulsant, ${ }^{4}$ antimicrobial, ${ }^{5}$ and antitumor agents. ${ }^{6}$

Published preparations of thiosemicarbazides 1 (Scheme 1) include (i) reactions of isothiocyanates with hydrazines, the method most frequently used, ${ }^{7 \mathrm{a}-\mathrm{e}}$ but isothiocyanates are difficult to handle and store; ${ }^{8 a-b}$ (ii) reduction of thiosemicarbazones by sodium borohydride is used for the preparation of and only applicable if $\mathrm{R}^{2}=\mathrm{H}$ mono-, di-, and tri- substituted $\mathbf{1}$ (but not tetra- or pentasubstituted); ${ }^{9}$ (iii) reactions of hydrazines with reactive thiocarbamic acid derivatives; the yields (66-73\%) were affected by side reactions; ${ }^{10-12}$ (iv) reactions of cyanohydrazines with hydrogen sulfide can yield mono or disubstituted thiosemicarbazides 1 $\left(\mathrm{R}^{1}=\mathrm{R}^{2}=\mathrm{H}\right){ }^{13}$ and (v) reactions of 1,2,4-triazolyl or bis(imidazolyl)methanethiones with amines then with hydrazines to give di- and trisubstituted thiosemicarbazides 1 . $^{14-15}$ 


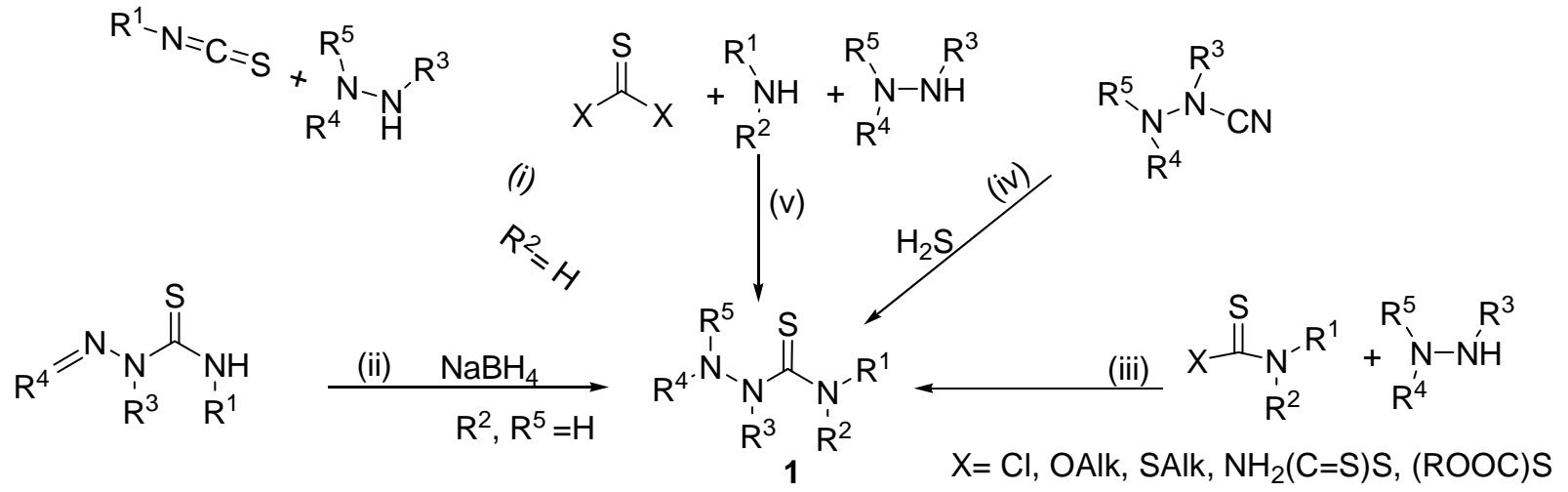

\section{Scheme 1}

Seventeen classes of $N$-substituted thiosemicarbazides can exist as shown in Table 1: three of mono (A, B, C), five of di (D through H), five of tri (I through $M)$, three of tetra $(\mathrm{N}, \mathrm{O}, \mathrm{P})$, and one of penta $(\mathrm{Q})$ substitution). Of these seventeen, nine have previously been reported in the literature (Table 1 classes A, B, C, D, E, F, G, H, M). While most of the other classes could potentially be made by one or more of the existing methods; a literature sub-structural search showed no known examples of compounds of classes I, J, K, L, N, O, P, Q.

Method $\mathrm{i}$ is convenient for the preparation of classes A, E, F. Methods ii and iii are mainly used for the preparation of class $F$ with a single example of class D using iii. Classes B, C, G, H, M are easily prepared using methods iv and $\mathrm{v}$ (Table 1). The work now presented provides an efficient route to the hither to unexplored classes $\mathrm{J}$ and $\mathrm{L}$ together with alternative access or potential access to classes A, B, C, E, F, G, H, M, P.

\section{$N$-Hydroxythioureas}

$N$-Hydroxythioureas are toxic to Lactobacillus arabinosus, Leuconostoc dextranicum and Streptococcus Faecalis, ${ }^{16}$ and some derivatives, e.g. S-methyl- $N$-hydroxyisothiourea, inhibit nitrous oxide synthase (NOS). ${ }^{17}$

Methods of preparation of $N$-hydroxythioureas 2 (Scheme 2) include (i) reactions of isothiocyanates with hydroxylamine to give $2(23-66 \%) ;^{16-20}$ (ii) the reduction of unstable $N, N^{`}-$ dihydroxythioureas. $^{21}$ 
Table 1. The possible classes of substituted thiosemicarbazides $\mathbf{1}$ and reported methods of preparation

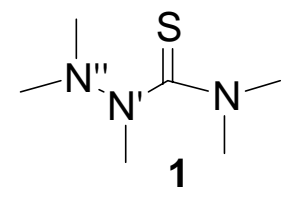

\begin{tabular}{|c|c|c|c|c|c|c|c|c|}
\hline \multicolumn{2}{|c|}{$N$-Substituents } & \multirow[t]{2}{*}{ Class } & \multicolumn{5}{|c|}{ Literature Methods (Scheme 1) } & \multirow{2}{*}{$\begin{array}{l}\text { This } \\
\text { work }\end{array}$} \\
\hline Number & Position & & $\mathbf{i}$ & ii & iii & iv & $\mathbf{v}$ & \\
\hline \multirow{3}{*}{ Mono } & $\mathbf{N}$ & A & $\mathrm{R}$ & $\mathrm{P}$ & $\mathrm{P}$ & - & $\mathrm{P}$ & $\mathrm{R}$ \\
\hline & $\mathbf{N}^{\prime}$ & $\mathbf{B}$ & - & $\mathrm{P}$ & - & $\mathrm{R}$ & $\mathrm{R}$ & $\mathrm{P}$ \\
\hline & $\mathbf{N}^{\prime \prime}$ & $\mathbf{C}$ & - & $\mathrm{P}$ & - & $\mathrm{R}$ & $\mathrm{R}$ & $\mathrm{P}$ \\
\hline \multirow{5}{*}{ Di } & NN & D & - & - & $\mathrm{P}$ & - & - & - \\
\hline & $\mathbf{N N}^{\prime}$ & $\mathbf{E}$ & $\mathrm{R}$ & $\mathrm{P}$ & $\mathrm{P}$ & - & $\mathrm{P}$ & $\mathrm{P}$ \\
\hline & NN" & $\mathbf{F}$ & $\mathrm{R}$ & $\mathrm{R}$ & $\mathrm{R}$ & - & $\mathrm{P}$ & $\mathrm{R}$ \\
\hline & N'N" & G & - & $\mathrm{P}$ & - & $\mathrm{R}$ & $\mathrm{R}$ & $\mathrm{P}$ \\
\hline & N'N"' & $\mathbf{H}$ & - & $\mathrm{P}$ & - & $\mathrm{R}$ & $\mathrm{R}$ & $\mathrm{P}$ \\
\hline \multirow{5}{*}{ Tri } & $\mathbf{N N N}^{\prime}$ & I & - & - & $\mathrm{P}$ & - & - & - \\
\hline & NN'N"' & $\mathbf{J}$ & $\mathrm{P}$ & $\mathrm{P}$ & $\mathrm{P}$ & - & $\mathrm{P}$ & $\mathrm{R}$ \\
\hline & NNN" & $\mathbf{K}$ & - & - & $\mathrm{P}$ & - & - & - \\
\hline & NN'N" & $\mathbf{L}$ & $\mathrm{P}$ & $\mathrm{P}$ & $\mathrm{P}$ & - & $\mathrm{P}$ & $\mathrm{R}$ \\
\hline & $\mathbf{N}^{\prime} \mathbf{N}^{\prime} \mathbf{N}^{\prime}$ & $\mathbf{M}$ & - & $\mathrm{P}$ & - & $\mathrm{R}$ & $\mathrm{R}$ & $\mathrm{P}$ \\
\hline \multirow{3}{*}{ Tetra } & NNN'N' & $\mathbf{N}$ & - & - & $\mathrm{P}$ & - & - & - \\
\hline & NNN"N" & $\mathbf{O}$ & - & - & $\mathrm{P}$ & - & - & - \\
\hline & NN'N"N"' & $\mathbf{P}$ & $\mathrm{P}$ & $\mathrm{P}$ & $\mathrm{P}$ & - & $\mathrm{P}$ & $\mathrm{P}$ \\
\hline Penta & NNN'N'N" & $\mathbf{Q}$ & - & - & $\mathrm{P}$ & - & - & - \\
\hline
\end{tabular}

R: Reported; P: Possible, but no example reported; -: Not possible 


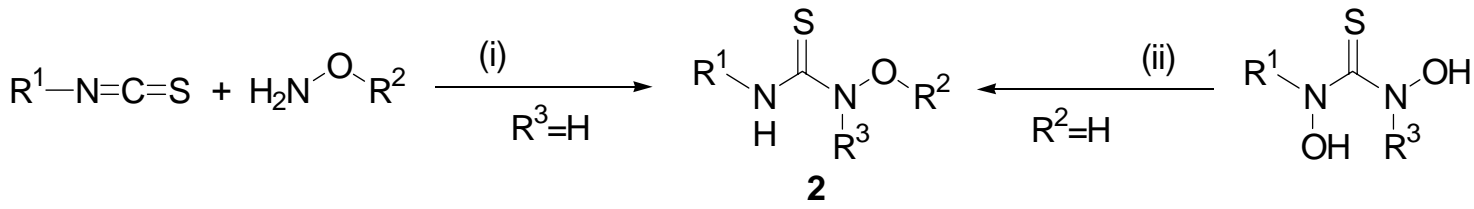

$$
\begin{aligned}
& \mathrm{R}^{1}, \mathrm{R}^{3}=\mathrm{Alk}, \mathrm{Ar} \\
& \mathrm{R}^{2}=\mathrm{H}, \mathrm{Alk}, \mathrm{Ar}
\end{aligned}
$$

\section{Scheme 2}

Eleven classes of $N, O$ substituted hydroxythioureas 2 can exist as shown in Table 2 ( three Mono, four Di, three Tri, and one Tetra). The two existing literature methods (i and ii), provide preparative methods for classes A', E', G' (Table 2). The present work affords an easy access to novel classes $\mathrm{C}^{\prime}, \mathrm{F}^{\prime}, \mathrm{H}^{\prime}$ in addition to A', B', E', G'. However, classes D', I', J', K', not previously reported, could not be prepared via our proposed procedure because although reacting 3 (Scheme 3) with secondary amines was successful, further reactions with hydrazines and hydroxylamines failed.

Table 2. The possible classes of substituted $N$-hydroxythioureas 2 and reported methods of preparation

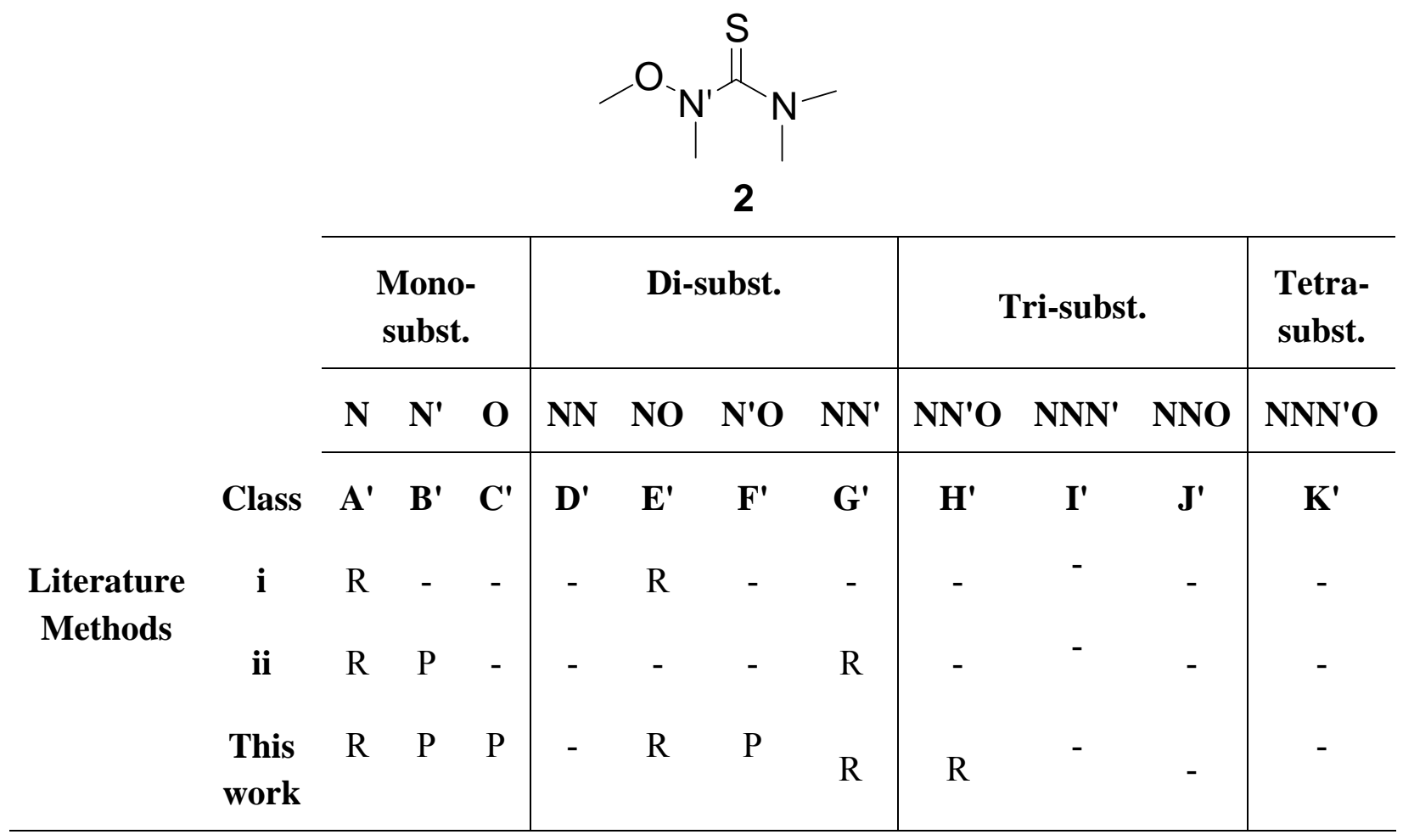

R: Reported; P: Possible but no example reported; -: not possible by this method 


\section{Results and Discussion}

Recently, we reported efficient synthesis of di- and trisubstituted thioureas 7 utilizing 1-(alkylor-arylthiocarbamoyl)benzotriazoles 4 (Scheme 3 ). ${ }^{22}$ We have now expanded this methodology to include the synthesis of thiosemicarbazides $\mathbf{5}$ and $\mathrm{N}$-hydroxythioureas $\mathbf{6}$.

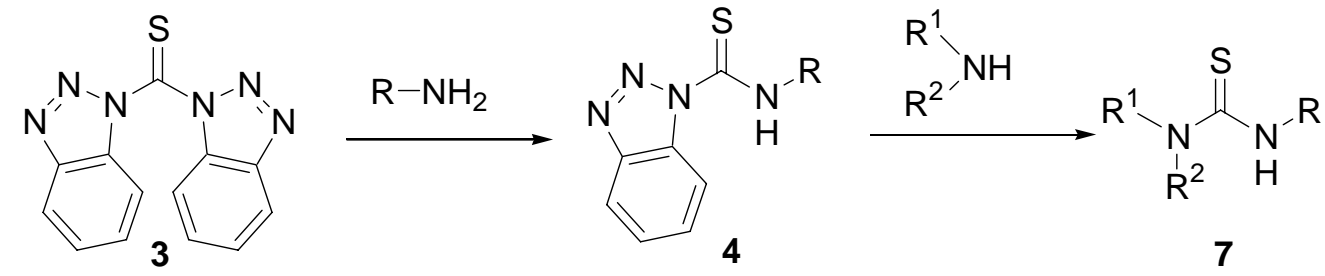

\section{Scheme 3}

Bis(benzotriazolyl)methanethione 3, a thiophosgene equivalent, is easily prepared from 1trimethylsilylbenzotriazole and thiophosgene in quantitative yield. ${ }^{23}$ As previously described, treatment of 3 with various primary amines in methylene chloride at room temperature followed by a $5 \% \quad \mathrm{Na}_{2} \mathrm{CO}_{3}$ wash and recrystallization afforded 1-(alkyl-orarylthiocarbamoyl)benzotriazoles $\mathbf{4 a - i}$ in $90-98 \%$ yields (Scheme 4 ). ${ }^{22}$ Only primary amines were used in the preparation of $\mathbf{4 a - i}$.

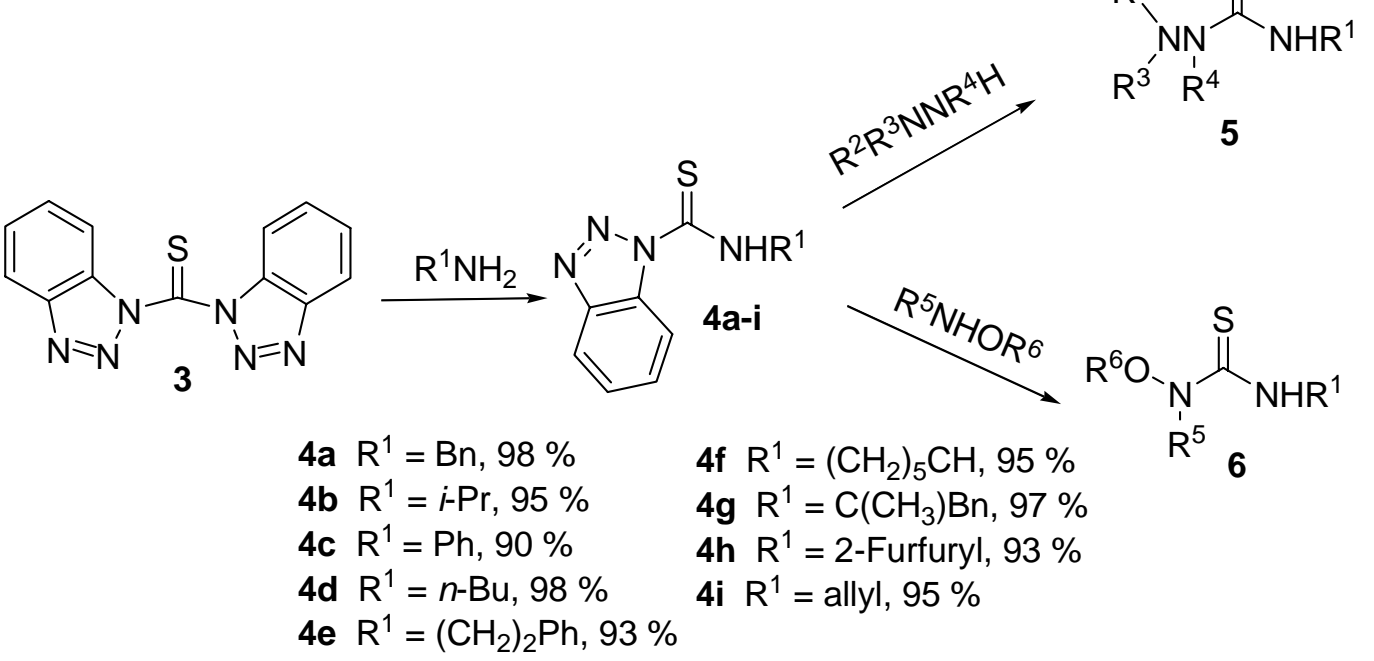

Structures $\mathbf{5}$ and $\mathbf{6}$ are fully defined in Tables 3 and 4 respectively

\section{Scheme 4}


Substituted thiosemicarbazides 5 were prepared via a single step reaction of 1-(alkyl-orarylthiocarbamoyl)benzotriazoles $\mathbf{4 a - i}$ with the appropriate hydrazine (Scheme 4, Table 3). Stirring 1 equiv. of 4 in methylene chloride at room temperature with 1.1 equiv. of the hydrazine and 2 equiv. of triethylamine followed by a $5 \% \mathrm{Na}_{2} \mathrm{CO}_{3}$ wash afforded 5 in excellent yields (Table 3). The reaction reached completion after $2 \mathrm{~h}$. as monitored by TLC. Substituted thiosemicarbazides $\mathbf{5}$ were purified using column chromatography (EtOAc/Hex) and characterized using NMR $\left({ }^{1} \mathrm{H},{ }^{13} \mathrm{C}\right.$ ). Melting points for known 5 agreed with reported values (see the Experimental Section). Novel 5 were characterized by ${ }^{1} \mathrm{H},{ }^{13} \mathrm{C}$ NMR spectra and elemental analyses (see the Experimental Section).

$N$-Hydroxythioureas 6 were prepared from the reaction of 1-(alkyl-orarylthiocarbamoyl)benzotriazoles $\mathbf{4 a - i}$ in methylene chloride at room temperature with 1.5 equiv. of the corresponding hydroxylamine and 3 equiv. of triethylamine (Scheme 4, Table 4). Starting materials disappeared completely after 5-12 h. as monitored by TLC. Formation of a white precipitate (benzotriazole triethylamine salt) marked the completion of the reaction. The precipitate was filtered and the filtrate washed with $5 \% \mathrm{Na}_{2} \mathrm{CO}_{3}$. The organic layer was extracted with methylene chloride (3 times), evaporated under vacuum, and chromatographed (EtOAc/Hex) to give $N$-hydroxythioureas 6 in excellent yields (Table 4). $N$-Hydroxythioureas 6 were fully characterized using NMR $\left({ }^{1} \mathrm{H},{ }^{13} \mathrm{C}\right)$ and elemental analysis. Melting points for known 6 agreed with reported values (see the Experimental Section). Novel 6 were characterized by ${ }^{1} \mathrm{H}$, ${ }^{13} \mathrm{C}$ NMR spectra and elemental analyses (see the Experimental Section).

Table 3. Preparation of substituted and unsubstituted thiosemicarbazides

\begin{tabular}{cccccc}
\hline $\mathrm{R}^{1}$ & $\mathrm{R}^{2}$ & $\mathrm{R}^{3}$ & $\mathrm{R}^{4}$ & Product & Yield\% \\
\hline $\mathrm{Cy}$ & $\mathrm{Ph}$ & $\mathrm{H}$ & $\mathrm{H}$ & $\mathbf{5 f}-\mathbf{a}$ & 88 \\
$n-\mathrm{Bu}$ & $\mathrm{Ph}$ & $\mathrm{H}$ & $\mathrm{H}$ & $\mathbf{5 d - a}$ & 85 \\
Phenethyl & $\mathrm{H}$ & $\mathrm{H}$ & $\mathrm{H}$ & $\mathbf{5 e - a}$ & 85 \\
$\mathrm{Cy}$ & $\mathrm{H}$ & $\mathrm{H}$ & $\mathrm{H}$ & $\mathbf{5 f}-\mathbf{b}$ & 91 \\
$\mathrm{Bn}$ & $\mathrm{H}$ & $\mathrm{H}$ & $\mathrm{H}$ & $\mathbf{5 a}-\mathbf{a}$ & 97 \\
Allyl & $\mathrm{H}$ & $\mathrm{H}$ & $\mathrm{H}$ & $\mathbf{5 i}-\mathbf{a}$ & 74 \\
DL-alpha-Methylbenzyl & $\mathrm{Me}$ & $\mathrm{Me}$ & $\mathrm{H}$ & $\mathbf{5 g}-\mathbf{a}$ & 50 \\
$n-\mathrm{Bu}$ & $\mathrm{Me}$ & $\mathrm{Me}$ & $\mathrm{H}$ & $\mathbf{5 d}-\mathbf{b}$ & 85 \\
Furfuryl & $\mathrm{Me}$ & $\mathrm{Me}$ & $\mathrm{H}$ & $\mathbf{5 h}-\mathbf{a}$ & 83 \\
$i-\mathrm{Pr}$ & $\mathrm{Me}$ & $\mathrm{H}$ & $\mathrm{Me}$ & $\mathbf{5 b}-\mathbf{a}$ & 78 \\
$\mathrm{Bn}$ & $\mathrm{Me}$ & $\mathrm{H}$ & $\mathrm{Me}$ & $\mathbf{5 a}-\mathbf{b}$ & 97 \\
\hline
\end{tabular}

* Solvent: $\mathrm{CH}_{2} \mathrm{Cl}_{2}$, Temp.: $25^{\circ} \mathrm{C}$, Time: $2 \mathrm{~h}$. 
Table 4. Preparation of substituted and unsubstituted $N$-hydroxythioureas

\begin{tabular}{ccccc}
\hline $\mathrm{R}^{1}$ & $\mathrm{R}^{5}$ & $\mathrm{R}^{6}$ & Product & Yield\% \\
\hline $\mathrm{Bn}$ & $\mathrm{H}$ & $\mathrm{H}$ & $\mathbf{6 a}-\mathbf{a}$ & 90 \\
$n-\mathrm{Bu}$ & $\mathrm{H}$ & $\mathrm{H}$ & $\mathbf{6 d}-\mathbf{a}$ & 77 \\
$\mathrm{Cy}$ & $\mathrm{H}$ & $\mathrm{H}$ & $\mathbf{6 f}-\mathbf{a}$ & 83 \\
$\mathrm{Ph}$ & $\mathrm{H}$ & $\mathrm{H}$ & $\mathbf{6 c}-\mathbf{a}$ & 99 \\
$i-\mathrm{Pr}$ & $\mathrm{Me}$ & $\mathrm{Me}$ & $\mathbf{6 b}-\mathbf{a}$ & 99 \\
$n-\mathrm{Bu}$ & $\mathrm{Me}$ & $\mathrm{H}$ & $\mathbf{6 d}-\mathbf{b}$ & 87 \\
DL-alpha-Methylbenzyl & $\mathrm{Me}$ & $\mathrm{Me}$ & $\mathbf{6 g}-\mathbf{a}$ & 89 \\
$i-\mathrm{Pr}$ & $\mathrm{H}$ & $\mathrm{Bn}$ & $\mathbf{6 b}-\mathbf{b}$ & 75 \\
Phenethyl & $\mathrm{H}$ & $\mathrm{Bn}$ & $\mathbf{6 e}-\mathbf{a}$ & 71 \\
\hline
\end{tabular}

* Solvent: $\mathrm{CH}_{2} \mathrm{Cl}_{2}$, Temp.: $25^{\circ} \mathrm{C}$, Time: 5-12h.

\section{Conclusion}

A new route providing easy access to thiosemicarbazides and $N$-hydroxythioureas of diverse substitution patterns in excellent yields has been established. Tables 1 and 2 compare our method to already reported routes of preparation of thiosemicarbazides and $N$-hydroxythioureas. Our method is particularly advantageous for the preparation of trisubstituted thiosemicarbazides (Table 1) and mono and disubstituted $N$-hydroxythioureas (Table 2). It is efficient with relatively short reaction time, and avoids the use of unstable isothiocyanates which are the classical starting materials for preparation of thiosemicarbazides and $N$-hydroxythioureas.

\section{Experimental Section}

General Procedures. Melting points were determined on a hot-stage apparatus and are uncorrected. NMR spectra were recorded in $\mathrm{CDCl}_{3}$, or DMSO- $d_{6}$ with TMS as the internal standard for ${ }^{1} \mathrm{H}(300 \mathrm{MHz})$ or a solvent as the internal standard for ${ }^{13} \mathrm{C}$ NMR $(75 \mathrm{MHz})$. Column chromatography was conducted on silica gel (200-425 mesh). Bis-benzotriazol-1-ylmethanethione 3 was prepared according to a previously reported procedure; $\mathrm{Mp} 171-172^{\circ} \mathrm{C}$, yield $98 \%$, (Lit. Mp $170-171{ }^{\circ} \mathrm{C}$, yield $90 \%$ ). ${ }^{23}$

General procedure for the preparation of $\mathbf{4 a - i}$. 1-Thiocarbamoyl benzotriazoles $\mathbf{4 a - i}$ were synthesized by the reaction of compound $3(2 \mathrm{mmol})$ and the appropriate primary amine $(2$ $\mathrm{mmol}$ ) in methylene chloride at room temperature for $2 \mathrm{~h}$ according to reported procedure. ${ }^{22}$ Melting points and spectral data were used to characterize known 4a-f, h-i and were found to be identical to reported values: $\mathbf{4 a} \mathrm{mp} 108-109{ }^{\circ} \mathrm{C}$ (Lit. mp 108-109 ${ }^{\circ} \mathrm{C}$ ) ${ }^{22} \mathbf{4 b} \mathrm{mp} 107-108{ }^{\circ} \mathrm{C}$ (Lit. mp 107. $7^{\circ} \mathrm{C}$ ); ${ }^{24} \mathbf{4 c ~ m p ~} 98-99{ }^{\circ} \mathrm{C}$ (Lit. mp $98.5^{\circ} \mathrm{C}$ ); ${ }^{24} \mathbf{4 d} \mathrm{mp} 92-93{ }^{\circ} \mathrm{C}$ (Lit. mp $92.3^{\circ} \mathrm{C}$ ); ${ }^{24} \mathbf{4 e} \mathrm{mp}$ 
$110.5^{\circ} \mathrm{C}$ (Lit. mp $110.2^{\circ} \mathrm{C}$ ); ${ }^{24}$ 4f mp $72{ }^{\circ} \mathrm{C}$ (Lit. mp $72-73{ }^{\circ} \mathrm{C}$ ) $;^{22}$ 4h mp $117^{\circ} \mathrm{C}$ (Lit. mp 117-119 $\left.{ }^{\circ} \mathrm{C}\right){ }^{22} \mathbf{4 i} \mathrm{mp} 56.4^{\circ} \mathrm{C}$ (Lit. mp 56-57 ${ }^{\circ} \mathrm{C}$ ). ${ }^{22}$ Known $\mathbf{4 g}$ was isolated as a yellow oil; ${ }^{22}$ spectral data and elemental analysis were used for characterization.

General procedure for the preparation of compounds 5. To a stirred solution of (1.15 mmol) $4 \mathbf{a}-\mathbf{i}$ in $12 \mathrm{ml}$ of dichloromethane, was added $(1.27 \mathrm{mmol})$ of the corresponding hydrazine hydrate followed by $(2.5 \mathrm{mmol})$ of triethylamine. The mixture was stirred for 3 hours at room temperature, then $10 \mathrm{ml}$ of $5 \% \mathrm{Na}_{2} \mathrm{CO}_{3}$ were added to remove excess benzotriazole. The solution was extracted with dichloromethane $(3 \times 50 \mathrm{ml})$ and the organic layer was dried over magnesium sulfate. Evaporating the solvent under reduced pressure followed by column chromatography (EtOAc/Hex gradient) afforded pure 5 in 50-97\% yield.

$\mathbf{N}$-Cyclohexyl-2-phenyl-1-hydrazinecarbothioamide (5f-a). ${ }^{25}$ Recrystallized from EtOAc/Hex to give pink crystals $(88 \%)$, mp $165^{\circ} \mathrm{C}$ (lit. $\left.163^{\circ} \mathrm{C}\right) ;{ }^{1} \mathrm{H}$ NMR $\delta 7.32-7.26(\mathrm{~m}, 2 \mathrm{H}), 7.19$ (br s, $1 \mathrm{H}), 7.12-7.10(\mathrm{~m}, 1 \mathrm{H}), 6.97-7.02(\mathrm{~m}, 1 \mathrm{H}), 6.81-6.86(\mathrm{~m}, 2 \mathrm{H}), 5.71$ (br s, $1 \mathrm{H}), 4.27-4.24(\mathrm{~m}$, $1 \mathrm{H}), 2.06-2.03(\mathrm{~m}, 2 \mathrm{H}), 1.72-1.61(\mathrm{~m}, 3 \mathrm{H}), 1.42-1.34(\mathrm{~m}, 2 \mathrm{H}), 1.23-1.11(\mathrm{~m}, 3 \mathrm{H}) ;{ }^{13} \mathrm{C} \mathrm{NMR} \delta$ $146.1,134.8,129.6,122.4,113.5,53.0,32.7,25.4,24.8$.

N-Butyl-2-phenyl-1-hydrazinecarbothioamide (5d-a). ${ }^{12}$ Oil $(85 \%) ;{ }^{1} \mathrm{H}$ NMR $\delta 7.48$ (br s, $1 \mathrm{H}), 7.30-7.23(\mathrm{~m}, 3 \mathrm{H}), 6.95-7.01(\mathrm{~m}, 1 \mathrm{H}), 6.92-6.86(\mathrm{~m}, 2 \mathrm{H}), 5.89$ (br s, $1 \mathrm{H}), 3.63$ (q, $J=7.1$ $\mathrm{Hz}, 2 \mathrm{H}), 1.59-1.54(\mathrm{~m}, 2 \mathrm{H}), 1.37-1.29(\mathrm{~m}, 2 \mathrm{H}), 0.91(\mathrm{t}, J=7.3 \mathrm{~Hz}, 3 \mathrm{H}) ;{ }^{13} \mathrm{C} \mathrm{NMR} \delta 146.1$, $129.5,129.3,122.3,113.5,44.1,31.1,19.9,13.7$.

$\mathbf{N}$-Phenethyl-1-hydrazinecarbothioamide (5e-a). ${ }^{12}$ Recrystallized from EtOAc/Hex to give white prisms $(85 \%), \mathrm{mp} 115^{\circ} \mathrm{C}$ (lit. $\left.113{ }^{\circ} \mathrm{C}\right) ;{ }^{1} \mathrm{H}$ NMR $\delta 8.13(\mathrm{~s}, 1 \mathrm{H}), 7.49$ (br s, 1H), $7.33-7.27$ (m, 2H), 7.24-7.22 (m, 3H), 3.89-3.83 (m, 2H), $3.77(\mathrm{~s}, 2 \mathrm{H}), 2.94-2.89(\mathrm{~m}, 2 \mathrm{H}) ;{ }^{13} \mathrm{C}$ NMR (DMSO) $\delta$ 158.7, 128.7, 128.4, 128.3, 126.1, 36.2, 34.9.

$\mathbf{N}$-Cyclohexyl-1-hydrazinecarbothioamide (5f-b). ${ }^{27}$ Recrystallized from EtOAc/Hex to give white crystals $(91 \%)$, mp $142{ }^{\circ} \mathrm{C}$ (lit. $\left.143{ }^{\circ} \mathrm{C}\right)$; ${ }^{1} \mathrm{H}$ NMR $\delta 7.34$ (br s, 1H), 7.19 (br s, 1H), 4.26$4.20(\mathrm{~m}, 1 \mathrm{H}), 3.71(\mathrm{~s}, 2 \mathrm{H}), 2.08-2.03(\mathrm{~m}, 2 \mathrm{H}), 1.77-1.71(\mathrm{~m}, 2 \mathrm{H}), 1.66-1.60(\mathrm{~m}, 2 \mathrm{H}), 1.46-1.36$ (m, 2H), 1.30-1.18 (m, 2H); ${ }^{13} \mathrm{C}$ NMR $\delta$ 152.4, 52.6, 32.9, 25.5, 24.8.

$\mathrm{N}$-Benzyl-1-hydrazinecarbothioamide (5a-a). ${ }^{27}$ Recrystallized from $\mathrm{CH}_{2} \mathrm{Cl}_{2} / \mathrm{Hex}$ to give colorless plates (97\%), mp $125-127{ }^{\circ} \mathrm{C}$ (lit. 126-128 ${ }^{\circ} \mathrm{C}$ ); ${ }^{1} \mathrm{H}$ NMR $\delta 7.97$ (s, 1H), 7.73 (br s, 1H), 7.35-7.28 (m, 5H), $4.84(\mathrm{~d}, J=5.8 \mathrm{~Hz}, 2 \mathrm{H}), 3.78$ (br s, $2 \mathrm{H}) ;{ }^{13} \mathrm{C}$ NMR $\delta 182.3,137.8$, 128.6, 127.7, 127.6, 47.8.

N-Allyl-1-hydrazinecarbothioamide (5i-a). ${ }^{28}$ Recrystallized from $\mathrm{CH}_{2} \mathrm{Cl}_{2} / \mathrm{Hex}$ to give colorless microcrystals (74\%), mp 93- $94{ }^{\circ} \mathrm{C}$ (lit. 90- $94{ }^{\circ} \mathrm{C}$ ); ${ }^{1} \mathrm{H}$ NMR $\delta 8.07$ (s, 1H), 7.54 (br s, $1 \mathrm{H}), 5.99-5.86(\mathrm{~m}, 1 \mathrm{H}), 5.27(\mathrm{~d}, \mathrm{~J}=1.4 \mathrm{~Hz}, 0.5 \mathrm{H}), 5.22-5.20(\mathrm{~m}, 1 \mathrm{H}), 5.16(\mathrm{~d}, \mathrm{~J}=1.4 \mathrm{~Hz}, 0.5 \mathrm{H})$, $4.29(\mathrm{t}, \mathrm{J}=5.9 \mathrm{~Hz}, 2 \mathrm{H}), 3.87$ (br s, $2 \mathrm{H}) ;{ }^{13} \mathrm{C}$ NMR $\delta 182.0,133.7,116.6,46.3$.

2,2-Dimethyl-N-(DL-alpha-methylbenzyl)-1-hydrazinecarbothioamide $\quad$ (5g-a). 12 Recrystallized from EtOAc/Hex to give white crystals $(50 \%), \mathrm{mp} 105-107{ }^{\circ} \mathrm{C}$ (lit. $\left.105{ }^{\circ} \mathrm{C}\right) ;{ }^{1} \mathrm{H}$ NMR $\delta 7.6$ (br s, 1H), 7.36-7.35 (m, 4H), 7.30-7.26 (m, 1H), 6.59 (br s, 1H), 5.69-5.64 (m, $1 \mathrm{H}), 2.56(\mathrm{~s}, 3 \mathrm{H}), 2.53(\mathrm{~s}, 3 \mathrm{H}), 1.59(\mathrm{~s}, 1 \mathrm{H}), 1.61(\mathrm{~s}, 1 \mathrm{H}) ;{ }^{13} \mathrm{C} \mathrm{NMR} \delta 142.8,135.5,128.6,127.3$, $126.2,52.6,47.3,47.1,21.6$. 
N-Butyl-2,2-dimethyl-1-hydrazinecarbothioamide (5d-b). ${ }^{12}$ Oil (85\%); ${ }^{1} \mathrm{H}$ NMR $\delta 7.23$ (br s, $1 \mathrm{H}), 6.25$ (br s, 1H), 3.67-3.60 (m, 2H), 2.53 (s, 6H), 1.66-1.57 (m, 2H), 1.43-1.35 (m, 2H), $0.96(\mathrm{t}, J=7.3 \mathrm{~Hz}, 3 \mathrm{H}) ;{ }^{13} \mathrm{C} \mathrm{NMR} \delta 158.0,47.2,43.8,31.3,20.1,13.8$.

N- (2-Furylmethyl)-2,2-dimethyl-1-hydrazinecarbothioamide (5h-a). Recrystallized from EtOAc/Hex to give colorless rods $(83 \%), \mathrm{mp} 106{ }^{\circ} \mathrm{C} ;{ }^{1} \mathrm{H}$ NMR $\delta 7.57$ (br s, 1H), 7.39 (s, 1H), 7.02 (br s, 1H), 6.36-6.34 (m, 1H), 6.31-6.30 (m, 1H), $4.84(\mathrm{~d}, J=5.5 \mathrm{~Hz}, 2 \mathrm{H}), 2.54(\mathrm{~s}, 6 \mathrm{H}) ;{ }^{13} \mathrm{C}$ NMR $\delta 150.7,142.2,138.0,110.4,107.8,47.0,40.8$. Anal. Calcd for $\mathrm{C}_{8} \mathrm{H}_{13} \mathrm{~N}_{3} \mathrm{OS}$ : C, 48.22; $\mathrm{H}$, 6.58; N, 21.09. Found: C, 48.55; H, 6.77; N, 21.34.

$\boldsymbol{N}$-Isopropyl-1,2-dimethyl-1-hydrazinecarbothioamide $\quad(5 \mathbf{b}-\mathbf{a}) . \quad 29$ Recrystallized from $\mathrm{CH}_{2} \mathrm{Cl}_{2} / \mathrm{Hex}$ to give colorless needles (78\%), mp $64-65{ }^{\circ} \mathrm{C}$ (lit. 64.0 - $65.0^{\circ} \mathrm{C}$ ); ${ }^{1} \mathrm{H}$ NMR $\delta 7.65$ $(\mathrm{s}, 1 \mathrm{H}), 4.52-4.41(\mathrm{~m}, 1 \mathrm{H}), 3.53(\mathrm{~s}, 3 \mathrm{H}), 3.36(\mathrm{q}, J=5.6 \mathrm{~Hz}, 1 \mathrm{H}), 2.59(\mathrm{~d}, J=5.6 \mathrm{~Hz}, 3 \mathrm{H}), 1.22$ $(\mathrm{d}, J=6.5 \mathrm{~Hz}, 6 \mathrm{H}) ;{ }^{13} \mathrm{C}$ NMR $\delta 180.0,46.2,36.6,34.4,22.6$.

$\mathrm{N}$-Benzyl-1,2-dimethyl-1-hydrazinecarbothioamide (5a-b). ${ }^{30}$ Recrystallized from $\mathrm{CH}_{2} \mathrm{Cl}_{2 /} /$ Hex to give colorless cubes $(97 \%), \mathrm{mp} 116-117{ }^{\circ} \mathrm{C}$ (lit. $115-116^{\circ} \mathrm{C}$ ); ${ }^{1} \mathrm{H}$ NMR $\delta 8.06(\mathrm{~s}, 1 \mathrm{H})$, $7.33-7.24(\mathrm{~m}, 5 \mathrm{H}), 4.80(\mathrm{~d}, J=5.6 \mathrm{~Hz}, 2 \mathrm{H}), 3.55(\mathrm{~s}, 3 \mathrm{H}), 3.40-3.39(\mathrm{~m}, 1 \mathrm{H}), 2.57(\mathrm{~d}, J=5.6$ $\mathrm{Hz}, 3 \mathrm{H}) ;{ }^{13} \mathrm{C}$ NMR $\delta 181.5,138.3,128.5,127.5,127.3,48.8,36.9,34.5$.

General procedure for the preparation of compounds 6. To a stirred solution of (2.0 mmol) $4 \mathbf{a}-\mathbf{i}$ in $15 \mathrm{ml}$ of dichloromethane, was added $(3.0 \mathrm{mmol})$ of the corresponding hydroxylamine hydrochloride followed by $(9.0 \mathrm{mmol})$ of triethylamine. The mixture was stirred for 5 hours at room temperature. Completion of the reaction is marked by the formation of a white precipitate (benzotriazole triethylamine salt). The reaction mixture was evaporated under reduced pressure then redissolved in diethyl ether. The precipitate formed was filtered, followed by addition of 10 $\mathrm{ml}$ of $5 \% \mathrm{Na}_{2} \mathrm{CO}_{3}$ to remove excess benzotriazole. The solution was extracted with dichloromethane $(3 \times 50 \mathrm{ml})$ and the organic layer was dried over magnesium sulfate. Evaporating the solvent under reduced pressure followed by column chromatography (EtOAc/Hex gradient) afforded pure 6 in $71-99 \%$ yield.

$\boldsymbol{N}$-Benzyl- $\boldsymbol{N}$-hydroxythiourea (6a-a). ${ }^{16}$ Recrystallized from EtOAc/Hex to give white powder (90\%), mp $156^{\circ} \mathrm{C}$ (lit. 155-157 $\left.{ }^{\circ} \mathrm{C}\right) ;{ }^{1} \mathrm{H}$ NMR $\delta$ 7.26-7.22 (m, 3H), 7.19-7.15 (m, 2H), 6.05 (br s, $1 \mathrm{H}), 4.56$ (s, 2H), 1.54 (br s, $1 \mathrm{H}) ;{ }^{13} \mathrm{C}$ NMR $\delta 153.7,136.5,128.9,128.0,127.5,48.6$.

$\boldsymbol{N}$-Butyl- $\boldsymbol{N}$-hydroxythiourea (6d-a). ${ }^{16}$ Recrystallized from $\mathrm{CH}_{2} \mathrm{Cl}_{2} / \mathrm{Hex}$ to give colorless plates (77\%), mp $107{ }^{\circ} \mathrm{C}$ (lit. 107-108 ${ }^{\circ} \mathrm{C}$ ); ${ }^{1} \mathrm{H}$ NMR $\delta 5.90$ (br s, 1H), 3.42 (br s, 2H), 1.64-1.55 (m, 2H), $1.45-1.33(\mathrm{~m}, 2 \mathrm{H}), 0.95(\mathrm{t}, J=7.3 \mathrm{~Hz}, 3 \mathrm{H}) ;{ }^{13} \mathrm{C}$ NMR $\delta 181.3,44.0,31.0,20.0,13.7$.

$\boldsymbol{N}$-Cyclohexyl- $\boldsymbol{N}$-hydroxythiourea (6f-a). ${ }^{16}$ Recrystallized from EtOAc/Hex to give brown powder (83\%), mp $116{ }^{\circ} \mathrm{C}$ (lit 116-118 ${ }^{\circ} \mathrm{C}$ ); ${ }^{1} \mathrm{H}$ NMR $\delta 6.01$ (br s, $1 \mathrm{H}$ ), 4.53 (br s, $1 \mathrm{H}$ ), $3.49-3.45(\mathrm{~m}, 1 \mathrm{H}), 2.00-1.92(\mathrm{~m}, 2 \mathrm{H}), 1.74-1.60(\mathrm{~m}, 3 \mathrm{H}), 142-1.06(\mathrm{~m}, 5 \mathrm{H}) ;{ }^{13} \mathrm{C} \mathrm{NMR} \delta$ $158.2,49.4,33.6,25.5,24.8$.

$\boldsymbol{N}$-Hydroxy- $\boldsymbol{N}$-phenylthiourea (6c-a). ${ }^{16}$ Recrystallized from $\mathrm{CH}_{2} \mathrm{Cl}_{2} / \mathrm{Hex}$ to give white microcrystals $(99 \%), \mathrm{mp} 103{ }^{\circ} \mathrm{C}$ (lit. $103{ }^{\circ} \mathrm{C}$ ); ${ }^{1} \mathrm{H}$ NMR $\delta 8.13$ (s, 1H), 7.43-7.36 (m, 5H), 7.30-7.25 (m, 1H); ${ }^{13} \mathrm{C}$ NMR $\delta 179.7,137.0,129.5,127.0,125.2$. 
$\boldsymbol{N}^{\prime}$-Isopropyl- $\boldsymbol{N}$-methoxy- $\boldsymbol{N}$-methylthiourea (6b-a). Colorless liquid (99\%); ${ }^{1} \mathrm{H} \mathrm{NMR} \delta 6.74$ $(\mathrm{s}, 1 \mathrm{H}), 4.59-4.43(\mathrm{~m}, 1 \mathrm{H}), 3.70(\mathrm{~s}, 3 \mathrm{H}), 3.55(\mathrm{~s}, 3 \mathrm{H}), 1.26(\mathrm{~d}, J=6.6 \mathrm{~Hz}, 6 \mathrm{H}) ;{ }^{13} \mathrm{C} \mathrm{NMR} \delta$ 180.0, 60.4, 46.2, 38.0, 22.2. Anal. Calcd for $\mathrm{C}_{6} \mathrm{H}_{14} \mathrm{~N}_{2} \mathrm{OS}$ : C, 44.42; H, 8.70; N, 17.27. Found: C, 44.79; H, 8.74; N, 17.13.

$\boldsymbol{N}$-Butyl- $\boldsymbol{N}$-hydroxy- $\boldsymbol{N}$-methylthiourea (6d-b). Oil (87\%); ${ }^{1} \mathrm{H}$ NMR $\delta 7.90$ (br s, 1H), 6.99 (br $\mathrm{s}, 1 \mathrm{H}), 3.61(\mathrm{~s}, 3 \mathrm{H}), 3.55(\mathrm{q}, J=7.1 \mathrm{~Hz}, 2 \mathrm{H}), 1.62-1.54(\mathrm{~m}, 2 \mathrm{H}), 1.42-1.35(\mathrm{~m}, 2 \mathrm{H}), 0.95(\mathrm{t}, J=$ $7.4 \mathrm{~Hz}, 3 \mathrm{H}) ;{ }^{13} \mathrm{C}$ NMR $\delta$ 157.3, 44.9, 42.0, 31.3, 20.0, 13.8. Anal. Calcd for $\mathrm{C}_{6} \mathrm{H}_{14} \mathrm{~N}_{2} \mathrm{OS}: \mathrm{C}$, 44.42; H, 8.70; N, 17.27. Found: C, 44.75; H, 9.17; N, 17.52.

$\boldsymbol{N}$-Methoxy- $\boldsymbol{N}$-methyl- $\boldsymbol{N}$ '-(DL-alpha-methylbenzyl)thiourea (6g-a). Colorless oil $(89 \%) ;{ }^{1} \mathrm{H}$ NMR $\delta 7.36-7.34(\mathrm{~m}, 4 \mathrm{H}), 7.29-7.25(\mathrm{~m}, 1 \mathrm{H}), 7.08$ (d, J=7.3 Hz, 1H), 5.68-5.58 (m, 1H), 3.67 (s, 3H), $3.56(\mathrm{~s}, 3 \mathrm{H}), 1.59$ (d, $J=6.9 \mathrm{~Hz}, 3 \mathrm{H}) ;{ }^{13} \mathrm{C} \mathrm{NMR} \delta$ 180.2, 142.6, 128.5, 127.3, 126.1, 60.7, 53.3, 38.1, 21.3. Anal. Calcd for $\mathrm{C}_{11} \mathrm{H}_{16} \mathrm{~N}_{2} \mathrm{OS}$ : C, 58.90; H, 7.19; N, 12.49. Found: C, 59.27; H, 7.35; N, 12.74 .

$\boldsymbol{N}$ - (Benzyloxy)- $\mathbf{N}$-isopropylthiourea (6b-b). Recrystallized from $\mathrm{CH}_{2} \mathrm{Cl}_{2} / \mathrm{Hex}$ to give white microcrystals (75\%), mp $60{ }^{\circ} \mathrm{C} ;{ }^{1} \mathrm{H}$ NMR $\delta 7.94$ (br s, $\left.1 \mathrm{H}\right), 7.387 .44(\mathrm{~m}, 5 \mathrm{H}), 6.46$ (br s, $\left.1 \mathrm{H}\right)$, $4.82(\mathrm{~s}, 2 \mathrm{H}), 4.46-4.35(\mathrm{~m}, 1 \mathrm{H}), 1.10(\mathrm{~d}, J=6.6 \mathrm{~Hz}, 6 \mathrm{H}) ;{ }^{13} \mathrm{C} \mathrm{NMR} \delta 181.4,134.9,129.4$, 129.3, 129.0, 78.5, 46.0, 22.0. Anal. Calcd for $\mathrm{C}_{11} \mathrm{H}_{16} \mathrm{~N}_{2} \mathrm{OS}$ : C, 58.90; H, 7.19; N, 12.49. Found: C, 59.10; H, 7.39; N, 12.83 .

$\boldsymbol{N}$ - (Benzyloxy)- $\boldsymbol{N}$-phenethylthiourea (6e-a). Recrystallized from $\mathrm{CH}_{2} \mathrm{Cl}_{2} / \mathrm{Hex}$ to give white microcrystals (71\%), mp 79- $80{ }^{\circ} \mathrm{C} ;{ }^{1} \mathrm{H}$ NMR $\delta 8.34(\mathrm{~s}, 1 \mathrm{H}), 7.37-7.30(\mathrm{~m}, 5 \mathrm{H}), 7.27-7.19(\mathrm{~m}$, $5 \mathrm{H}), 6.79(\mathrm{~s}, 1 \mathrm{H}), 4.71(\mathrm{~s}, 2 \mathrm{H}), 3.83(\mathrm{q}, J=6.9 \mathrm{~Hz}, 2 \mathrm{H}), 2.85(\mathrm{t}, J=6.9 \mathrm{~Hz}, 2 \mathrm{H}) ;{ }^{13} \mathrm{C} \mathrm{NMR} \delta$ $182.0,138.2,134.5,129.2,129.1,128.8,128.7,126.7,78.4,44.9,34.8$. Anal. Calcd for $\mathrm{C}_{16} \mathrm{H}_{18} \mathrm{~N}_{2} \mathrm{OS}$ : C, 67.10; H, 6.33; N, 9.78. Found: C, 66.96; H, 6.41; N, 9.71.

\section{References}

1. Kappel, J. C.; Yokum, T. S.; Barany, G. J. Comb. Chem. 2004, 6, 746.

2. Sherman, W. R. J. Org. Chem. 1961, 26, 88.

3. Jalilian, A.R.; Sattari, S.; Bineshmarvasti, M.; Shafiee, A.; Daneshtalab, M. Arch. Pharm. Pharm. Med. Chem. 2000, 333, 347.

4. Chapleo, C. B.; Myers, P. L.; Smith, A. C. B.; Stillings, M. R.; Tulloch, I. F.; Walter, D. S. J. Med. Chem. 1988, 31, 7.

5. Da Silva, E. F.; Canto-Cavalheiro, M. M.; Braz, V. R.; Cysne-Finkelstein, L.; Leon, L. L.; Echevarria A. Euro. J. Med. Chem. 2002, 37, 979.

6. Grynberg, N.; Santos, A.C.; Echevarria, A. Anti-cancer drugs 1997, 8, 88.

7. (a) Walter, W.; Voss, J.; Org. Comp. Sulf. Selen. Tell. 1979, 139. (b) Baxter, A.; Bennion, C.; Bent, J.; Boden, K.; Brough, S.; Cooper, A.; Kinchin, E.; Kindon, N.; Mcinally, T.; Mortimore, M.; Roberts, B.; Unitt, J. Bio. Med. Chem. Lett. 2003, 13, 2625. (c) Coburn, A. 
R.; Glennon, R. A. J. Med. Chem. 1974, 17, 1025. (d) Jacobsen, N.; Toelberg, J. Synthesis 1986, 561. (e) L'abbe, G.; Leurs, S.; Sannen, I.; Dehaen, W. Tetrahedron 1993, 49, 4439.

8. (a) Brandsma, L.; Nedolya, N. A. ARKIVOC 2001, (ix), 7. (b) Nedolya, N. A.; Brandsma, L.; Schlyakhtina, N. I.; Lazarev, I. M.; Albanov, A. I.; Zinchenko, S. V.; Klyba, L. V. ARKIVOC 2001, (ix), 12.

9. Yamamoto, I; Ikui, A; Muneharu, N.; Kotani, M.; Motoyoshiya, J.; Gotoh, H.; Matsuzaki, K. J. Chem. Soc. 1983, P(1), 2297.

10. Bazavova, M.; Dubenko, R. G.; Pellkis, P. S. Zhur. Org. Khim. 1979, 17, 171.

11. Farhanullah, Sil, D.; Tripathi, B. K.; Srivastava, A. K.; Ram, V. J. Bio. Med. Chem. Lett. 2004, 14, 2571.

12. Jensen, K. A.; Anthoni, U.; Kagi, B.; Larsen, C.; Pedersen, C. T. Acta Chem. Scand. 1968, 22, 1

13. Scott, E.S.; Zeller, E. E.; Audrieth, L. F. J. Org. Chem. 1954, 749.

14. Anthoni, U.; Larsen, C.; Nielsen, P. H. Acta Chem. Scand. 1967, 21, 2061.

15. Larsen, C.; Harpp, D. N. Phosphorus and Sulfur 1984, 19, 91.

16. Clifton, G.; Bryant, S. R.; Skinner, C. G. J. Med. Chem. 1970, 13, 377.

17. Ichimori, K.; Stuehr, D. J. J. Med. Chem. 1999, 42, 1842.

18. Anthoni, U.; Larsen, C.; Nielsen, P. H. Acta Chem. Scand. 1967, 21, 2061.

19. Sato, M.; Stammer, C. H. J. Med. Chem. 1976, 19, 336.

20. Le, V. D.; Wong, C. H. J. Org. Chem. 2000, 65, 2399.

21. Haugwitz, R. D. Liebigs Ann. Chem. 1970, 731, 171.

22. Katritzky, A. R.; Ledoux, S; Witek, R. M.; Nair, S. K. J. Org. Chem. 2004, 69, 2976.

23. Larsen, C.; Steliou, K.; Harpp, D. N. J. Org. Chem. 1978, 43, 337.

24. Katritzky, A. R.; Khashab, N. M.; Bobrov, S. Helv. Chim. Acta 2005, 88, 1664.

25. Sasse, K.; Wiss, H Liebigs Ann. Chem. 1970, 735, 158.

26. Jazwinski, J.; Krajewska, O. S. J. Molecular Structure 2004, 687, 23.

27. Tisler, M. Croatica Chem. Acta 1956, 28, 147.

28. Aoyagi, E. I. Patent 518340, 1984; Chem. Abstr. 1985, 102, 95652

29. Pfeiffer, W. D.; Dilk, E., Roßberg, H.; Langer, P. Synlett 2003, 15, 2392.

30. Walter, W.; Rohloff, C. Liebigs Ann. Chem. 1977, 3, 463. 